\title{
netz:regeln 2017: künstlich intelligent
}

\section{Am 16.11.2017 veranstalten der Bitkom und die Heinrich-Böll-Stiftung gemeinsam die Konferenz netz:regeln 2017 in Berlin. Veranstaltungsort ist die Heinrich-Böll-Stiftung, Schumannstraße 8, in Berlin. Beginn ist um 9:30 Uhr.}

Künstliche Intelligenz (KI) dringt immer weiter in Bereiche vor, die die Bürgerinnen und Bürger direkt betreffen. Sie wird die Art und Weise, wie Menschen arbeiten, lernen, kommunizieren, konsumieren und leben, über die kommenden Jahre grundlegend verändern. Gesellschaft, Wirtschaft und Staat stehen vor der Aufgabe, diesen Wandel aktiv zu gestalten. Es ist wichtig, Verantwortlichkeiten beim Einsatz von KI zu bestimmen, einen klaren Rechtsrahmen zu entwickeln und gezielte Forschungsförderung entsprechend ethischer Standards zu betreiben.

\section{Die Leitfragen der Konferenz 2017:}

- Wie ist der Entwicklungsstand in den Bereichen Mobilität, Sicherheit und Gesundheit?

- Welchen Anteil hat künstliche Intelligenz am digitalen Wandel?

- Wie wird künstliche Intelligenz den Arbeitsmarkt verändern?
- Vor welche gesellschaftlichen und demokratischen Herausforderungen stellt uns KI?

- Welche Rahmenbedingungen müssen wir schaffen, um Chancen von KI zu nutzen und Risiken adäquat zu begegnen?

Mit netz:regeln 2017 laden der Bundesverband Informationswirtschaft, Telekommunikation und Neue Medien e.V. (Bitkom) und die Heinrich-Böll-Stiftung e.V. Expertinnen und Experten aus Wissenschaft und Forschung, Netzgemeinde, Politik und digitaler Wirtschaft zum achten digitalpolitischen Dialog ein.

Der Eintritt ist frei, es wird um Anmeldung gebeten. Aufgrund einer Systemumstellung können Sie sich momentan nur per E-Mail anmelden: anmeldung@boell.de.

Hinweis: Die Veranstaltung wird als Livestream übertragen. Weitere Informationen und den Link zum Livestream finden Sie unter: http://calendar.boell.de/de/event/netzregeln-2017kuenstlich-intelligent



